\title{
Introduction to HICCS-53 Minitrack on Crowdsourcing and the Digital Workforce in the Gig Economy
}

\author{
Xuefei (Nancy) Deng \\ California State University, \\ Dominguez Hills \\ ndeng@csudh.edu
}

\author{
Sara Moussaswi \\ Carnegie Mellon University \\ smoussaw@andrew.cmu.edu
}

\author{
Joseph D. Taylor \\ California State University, \\ Sacramento \\ joseph.taylor@csus.edu
}

\section{Introduction}

The "gig" economy has been described as individuals engaging in contract labor arrangements as a supplement or alternative to traditional employment. The rapid growth of digital platforms that enable the gig-based contract relationships is one factor contributing to this emerging employment trend [1].

While the use of digital platforms to facilitate work is growing, it's less clear if the structures or effects of organizational practices differ between workers engaged in digital platforms when compared to traditional organizations. The extant literature has established that workers finding meaningful work opportunities and receiving accurate feedback regarding work outcomes fosters intrinsic motivations [2], however within digital platforms the conditions under which these actions are taken differ from traditional employment.

Digital platforms enable new ways to supervise and motivate workers. Platforms such as Amazon Mechanical Turk (MTurk), Uber, and TaskRabbit provide access to on-demand workers who can engage in tasks as a virtual workforce. This emerging crowd work environment is shaped by six essential characteristics, including on-demand virtual labor, open access to work, internet access to join the crowd, human tasks, modular technical architecture, and three stakeholders of crowdsourcer, crowdsourcee, and crowdsourcing intermediary [3].

Digital platforms provide capabilities to coordinate work and facilitate administrative tasks between sourcers and sourcees. The administrative affordances of digital platforms demonstrate the need to expand on traditional work design theory by integrating motivational, social, and work context characteristics. While the digital workforce brings new, important competencies to the workplace, it may also develop new identity and new ways of collaborating [4]. One of the compelling aspects of the "Digital Workforce and Crowdwork" are the unique benefits and opportunities that it provides to both workers and firms engaged in digital activities [1]. Moreover, the digitalization of work is transforming not just organizations and industries, but potentially the whole labor market [5], calling for updating and developing public policies governing the emerging, global digital work environments and managing the digital workforce.

\section{Scope}

This minitrack focuses on the spectrum of experiences and influences of digital work and digital workforce. Research of this minitrack lies at the intersections of multiple disciplines, namely Information Technology, Organization Science, Human Resource Management, and Behavioral Science. This minitrack publishes both theoretical and empirical studies addressing organizational, managerial, technical, and behavioral perspectives on digital workforce and crowd work.

Topics and issues presented in this minitrack include, but not limited to:

- Crowdsourcing, crowd worker and digital workforce

- Crowd worker participation and motivation

- Crowd worker community

- Emerging digital professions and professional development

- Employment relations in crowdsourcing

- Ethical issues in managing the digital workforce

- Global workforce in the crowdsourcing

- Psychological aspects of digital workplace (e.g., Technostress)

- Skill development and career pathways of digital workforce

- Work design and work environment in crowdsourcing 
- Crowdsourcing for microtasking

- Task design for crowd engagement

- Crowdsourcing contest design

- Institutional practices and policies for crowdsourcing

- Management and practice of work in hyperdigital environments

- New work routines and future work design

- Regulatory challenges of crowdsourcing

- Gig work and workers in the gig economy

- Gigs and task design

- Gig worker motivation

- Employment relations in the gig economy

- Community effects of a distributed digital workforce

- Job and career opportunities in the gig economy

- Organizational and regulatory challenges in the gig economy

- Psychological well-beings of the gig workers

- Work-life balance of the gig workers

\section{Summary of Articles}

This mini-track presents two papers in HICCS-53. We introduce them briefly below.

First, digital platforms that facilitate gig work use a wide array of technologies to help match workers and positions. Many of the tasks that are completed by managers within traditional work environments are algorithmically managed on digital platforms. In their research, Jabagi, Croteau and Audebrand [6] provide a new conceptual model for understanding how workers perceive organizational support through algorithmic oversite. Their paper, titled "Perceived Organizational Support in the Face of Algorithmic Management: A Conceptual Model," uses organizational support theory to explore the antecedents of gig work and proposes that by promoting perceptions of fairness and managing performance-based rewards, digital platforms may be perceived to provide organizational support to workers.

Second, the gig economy engages workers in a wide array of industries. Some scholars are beginning to examine the differences between how gig workers are motivated across industries. In their paper, titled "What drives the drivers? A qualitative perspective on what motivates the crowd delivery workforce," Asdecker and Zirkelbach [7] conduct qualitative interviews with 27 delivery drivers participating in a variety of digital platforms. A synthesis of these interviews is provided using the self-determination view. They find that the motivations of crowd delivery drivers differ from those expressed by gig workers participating in digital platforms that support other industries. The findings provide useful implications for understanding the variation of motivations of the digital workforce.

\section{Reference}

[1] Taylor, J., and K. D. Joshi. "Joining the Crowd: The Career Anchors of Information Technology Workers Participating in Crowdsourcing," Information Systems Journal, vol. 16, no. 2, 2019, pp. 641-673.

[2] Hackman, J. R., and G. R. Oldham. "Motivation through the Design of Work: Test of a Theory," Organizational Behavior and Human Performance, vol. 16 , no. 2 , 1979 , pp. 250-279.

[3] Deng, X. and K. D. Joshi. "Why Individuals Participate in Micro-task Crowdsourcing Work Environment: Revealing Crowdworkers' Perceptions," Journal of the Association for Information Systems, vol. 17, no. 10, 2016, pp. 711-736.

[4] Colbert, A., N. Yee, and G. George. "The Digital Workforce and the Workplace of the Future," Academy of Management Journal, vol. 59, no. 3, 2016, pp. 731-739.

[5] Deng, X., K. D. Joshi, and R. D. Galliers. "The Duality of Empowerment and Marginalization in Microtask Crowdsourcing: Giving Voice to the Less Powerful Through Value Sensitive Design," MIS Quarterly, vol. 40, no. 2, 2016, pp. 279-302.

[6] Jabagi, N., A. Croteau, and L. Audebrand. "Perceived Organizational Support in the Face of Algorithmic Management: A Conceptual Model," in Proceedings of the 53rd Hawaii International Conference on Systems Science (HICSS -53), January 7-10, 2020, Maui, Hawaii.

[7] Asdecker, B., and F. Zirkelbach. "What Drives the Drivers? A Qualitative Perspective on What Motivates the Crowd Delivery Workforce," in Proceedings of the 53rd Hawaii International Conference on Systems Science (HICSS -53), January 7-10, 2020, Maui, Hawaii. 